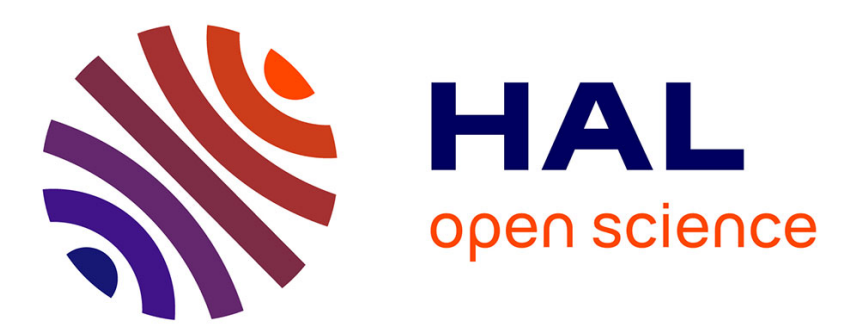

\title{
DÉTERMINATION DE VIES MOYENNES À L'AIDE D'UN CORRÉLATEUR DIGITAL EN TEMPS RÉEL
}

\author{
J. Danière, R. Rougny, E. Descroix, Daniel Charnay, Roland Billerey, Henri
}

Chevalier

\section{- To cite this version:}

J. Danière, R. Rougny, E. Descroix, Daniel Charnay, Roland Billerey, et al.. DÉTERMINATION DE VIES MOYENNES À L'AIDE D'UN CORRÉLATEUR DIGITAL EN TEMPS RÉEL. Nuclear Instruments and Methods, 1974, 115, pp.165-171. hal-01109240

\author{
HAL Id: hal-01109240 \\ https://hal.science/hal-01109240
}

Submitted on 26 Jan 2015

HAL is a multi-disciplinary open access archive for the deposit and dissemination of scientific research documents, whether they are published or not. The documents may come from teaching and research institutions in France or abroad, or from public or private research centers.
L'archive ouverte pluridisciplinaire HAL, est destinée au dépôt et à la diffusion de documents scientifiques de niveau recherche, publiés ou non, émanant des établissements d'enseignement et de recherche français ou étrangers, des laboratoires publics ou privés. 


\title{
DÉTERMINATION DE VIES MOYENNES À L'AIDE D'UN CORRÉLATEUR DIGITAL EN TEMPS RÉEL
}

\author{
J. DANIERE, R. ROUGNY, E. DESCROIX, D. CHARNAY et R. BILLEREY
}

Institut de Physique Nucléaire, Université Claude Bernard Lyon-I (Institut National de Physique Nucléaire et Physique des Particules) 43, Bd du 11 novembre 1918, 69621 Villeurbanne, France

\section{H. CHEVALIER}

Centre d'Études Nucléaires de Grenoble (L.E.T.I.), 38041 Grenoble, France

Received 16 July 1973 and in revised form 11 October 1973

A digital correlator giving the autocorrelation or the intercorrelation function of two signals $X(t)$ and $Y(t)$ in true time is used. The incremental delay is adjustable between $100 \mathrm{~ns}$ and $1 \mathrm{~s}$, by 100-ns steps.

\section{Introduction}

Les méthodes de corrélation présentent un grand intérêt pour l'extraction d'une information noyée dans un bruit. Des corrélateurs analogiques sont utilisés dans différents domaines ${ }^{1}$ ). En spectrométrie nucléaire une version digitalisée est mieux adaptée au caractère impulsionnel de l'information. Des travaux ont déjà été effectués dans ce sens pour des mesures de spectres de temps de $\mathrm{vol}^{2,3}$ ) et des mesures de vies moyennes longues ${ }^{4,5}$ ). Dans ce dernier domaine, nous utilisons un corrélateur digital qui, par le calcul en temps réel de l'intercorrélation de deux suites d'impulsions calibrées $X(t)$ et $Y(t)$ donne accès aux vies moyennes d'états métastables et au nombre de coïncidences vraies entre deux radiations passant par ces états.

Si les fonctions aléatoires $X(t)$ et $Y(t)$ sont stationnaires, leur fonction d'intercorrélation peut s'écrire :

$$
C_{\mathrm{XY}}(\lambda)=\operatorname{Lim}_{T \rightarrow \infty} \frac{1}{T} \int_{0}^{T} X(t) Y(t+\lambda) \mathrm{d} t,
$$

$T$ étant le temps de calcul.

Pour un système digitalisé, cette relation devient :

$$
C_{\mathrm{XY}}(\lambda)=\frac{1}{T} \sum_{i=0}^{T / \theta} X(i \theta) Y(i \theta+\lambda),
$$

$\theta$ étant le retard incrémental du corrélateur; $\lambda$ est alors un multiple de $\theta$.

\section{Description de l'appareil}

L'appareil réalisé dans le Service de Neutronique de 1'I.P.N. ${ }^{6}$ ) effectue en temps réel le calcul de la fonction
The application of this correlator to nuclear spectroscopy is studied and we compare different other methods used for nuclear lifetimes determination.

d'intercorrélation de deux signaux logiques $X$ et $Y$ avec une définition de 200 points. Le retard incrémental est réglable de $100 \mathrm{~ns}$ à $1 \mathrm{~s}$ par pas de $100 \mathrm{~ns}$.

\subsection{PRINCIPE}

Le corrélateur effectue les trois fonctions ci-dessous selon le schéma de la fig. 1.

1) Retard d'une voie (X) par pas de temps $\theta$.

2) Coïncidence entre les signaux de la voie $X$ retardée et les signaux de la voie $\mathrm{Y}$ pendant la durée $\theta$.

3) Comptage et mise en mémoire de ces coïncidences. Cet appareil a été réalisé en technologie TTL. Il doit être couplé avec une mémoire de stockage (utilisation d'un analyseur 400 canaux en mode multi-échelle par exemple).

\subsection{RETARD}

Le signal $\mathrm{X}$ à retarder est connecté directement à l'entrée d'un registre à décalage, ligne à retard digitale

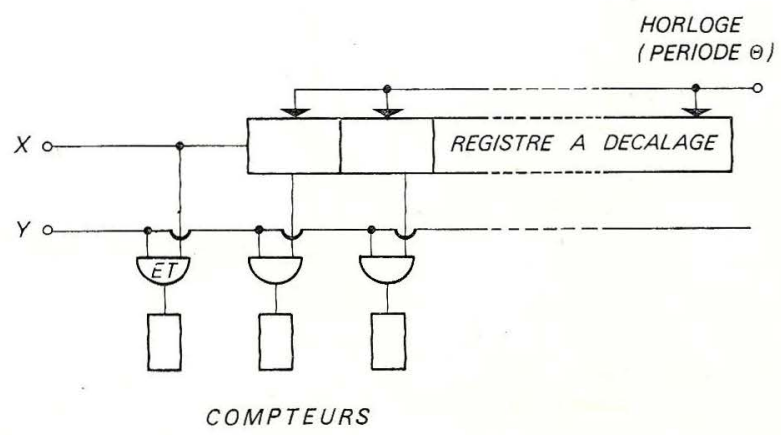

Fig. 1. Schéma de principe du corrélateur. 
constituée de bascules interconnectées en série. Le retard incrémental apporté à $\mathrm{X}$ d'une bascule à l'autre est égal à la période d'horloge $\theta$. Les différentes sorties des bascules donnent donc le signal $X$ aux instants, $t, t-\theta, t-2 \theta, \ldots$ L'information $\mathrm{X}$ n'est prise en compte à l'entrée du registre à décalage que si elle est présente au moment de l'impulsion d'horloge. Il faut donc lui imposer une largeur égale à $\theta$, ou la recaler de manière à la synchroniser avec l'impulsion d'horloge suivante (mise en phase). A la sortie de la première bascule du registre à décalage, l'impulsion $\mathrm{X}$ est quantifiée à un pas égal à $\theta$ et se propage ainsi dans tout le registre.

\subsection{COIINCIDENCES ENTRE LES VOIES X ET Y}

Les signaux étant des impulsions logiques, le circuit de coïncidence pour chaque retard se réduit à une porte ET.

Si l'impulsion Y chevauche un instant de décalage, elle peut être comptée dans deux canaux consécutifs, il faut donc: soit la rendre très étroite, soit la recaler entre deux impulsions d'horloge (mise en phase). C'est cette dernière solution qui est utilisée.

\subsection{Comptage et lecture}

Comme il est impensable de connecter à la sortie de chaque porte de corrélation un compteur de grande capacité, on s'est limité à un compteur de 8 bits par point. Chaque point est lu périodiquement et son contenu transféré dans la mémoire de stockage. La capacité maximum par point est donc : 255 impulsions.

\subsubsection{Lecture séquentielle des points de corrélation}

Les bits de même poids des 200 points de corrélation sont mis en parallèle par l'intermédiaire de portes ET à deux entrées, la deuxième entrée étant utilisée pour

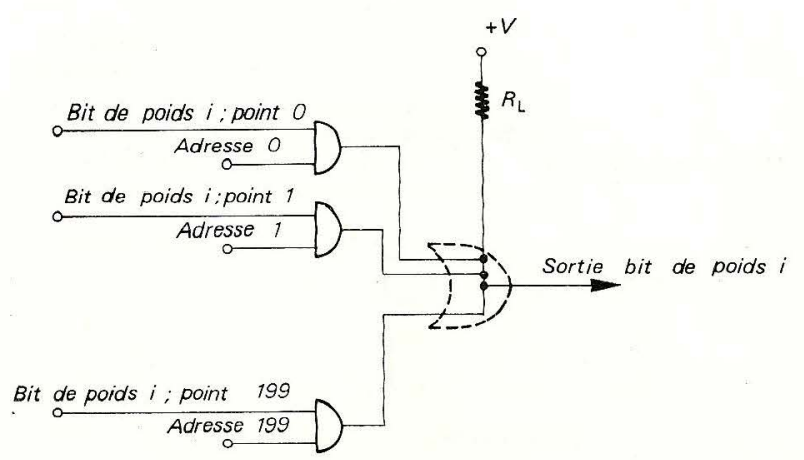

Fig. 2. Principe de la lecture du canal $i$. l'adresse du point (fig. 2), la sortie est du type collecteur ouvert et permet de réaliser un «OU cablé ».

Cette structure permet de lire de façon séquentielle la fonction de corrélation sur 8 fils représentant les 8 bits de définition de chaque point (fig. 3).

\subsubsection{Transfert du contenu des points}

A l'adresse d'un point on dispose donc d'un «mot binaire» correspondant aux 8 bits de définition de celui-ci. Si aucune impulsion ne s'est présentée à l'entrée des compteurs $600 \mathrm{~ns}$ (temps de propagation d'une impulsion de l'entrée du compteur au bit de poids le plus fort) avant l'ordre de stockage, le mot est transféré dans une mémoire tampon puis le compteur remis à zéro. Pendant le temps de transfert (200 ns) l'entrée $Y$ est bloquée (temps mort). On évite ainsi les risques d'erreurs dus aux changements d'état des compteurs pendant leur lecture.

On dispose donc de l'intervalle de temps séparant la mémorisation d'un point de la lecture du point suivant pour transférer le contenu de la mémoire tampon dans la mémoire de stockage. Cela est réalisé en convertissant le contenu de la mémoire tampon en un train d'impulsions dont le nombre est égal à la valeur du mot binaire qui était inscrit dans cette mémoire.

Le train d'impulsions série entre directement sur le registre arithmétique d'un sélecteur 400 canaux en mode multi-échelle, l'avance adresse et la remise à zéro adresse étant fournies par le corrélateur.

\subsubsection{Choix de la fréquence lecture}

Cette fréquence $f_{l}$ peut être choisie en fonction du type d'expérience. On a intérêt à la choisir aussi faible que possible pour diminuer le temps mort provoqué par le blocage de la voie $\mathrm{Y}$ pendant la lecture du contenu de chaque point de corrélation. La limite inférieure est imposée par la fréquence maximum $f_{y_{\max }}$ des coïncidences enregistrée à la sortie de l'une des 200 portes, alors :

$$
f_{l}>f_{y_{\max }} \frac{200}{255} .
$$

La limite supérieure de la fréquence de lecture est imposée par la mémoire de stockage (fréquence du train série $f_{\mathrm{s}}$ et fréquence avance adresse $f_{\mathrm{aa}}$ de l'analyseur 400 canaux utilisés) par l'une des deux conditions :

$\mathrm{ou}$

$$
f_{l}<f_{\mathrm{s}} / 255
$$

$$
f_{l}<f_{\mathrm{aa}} .
$$

La fig. 4 donne le diagramme des temps pour la 


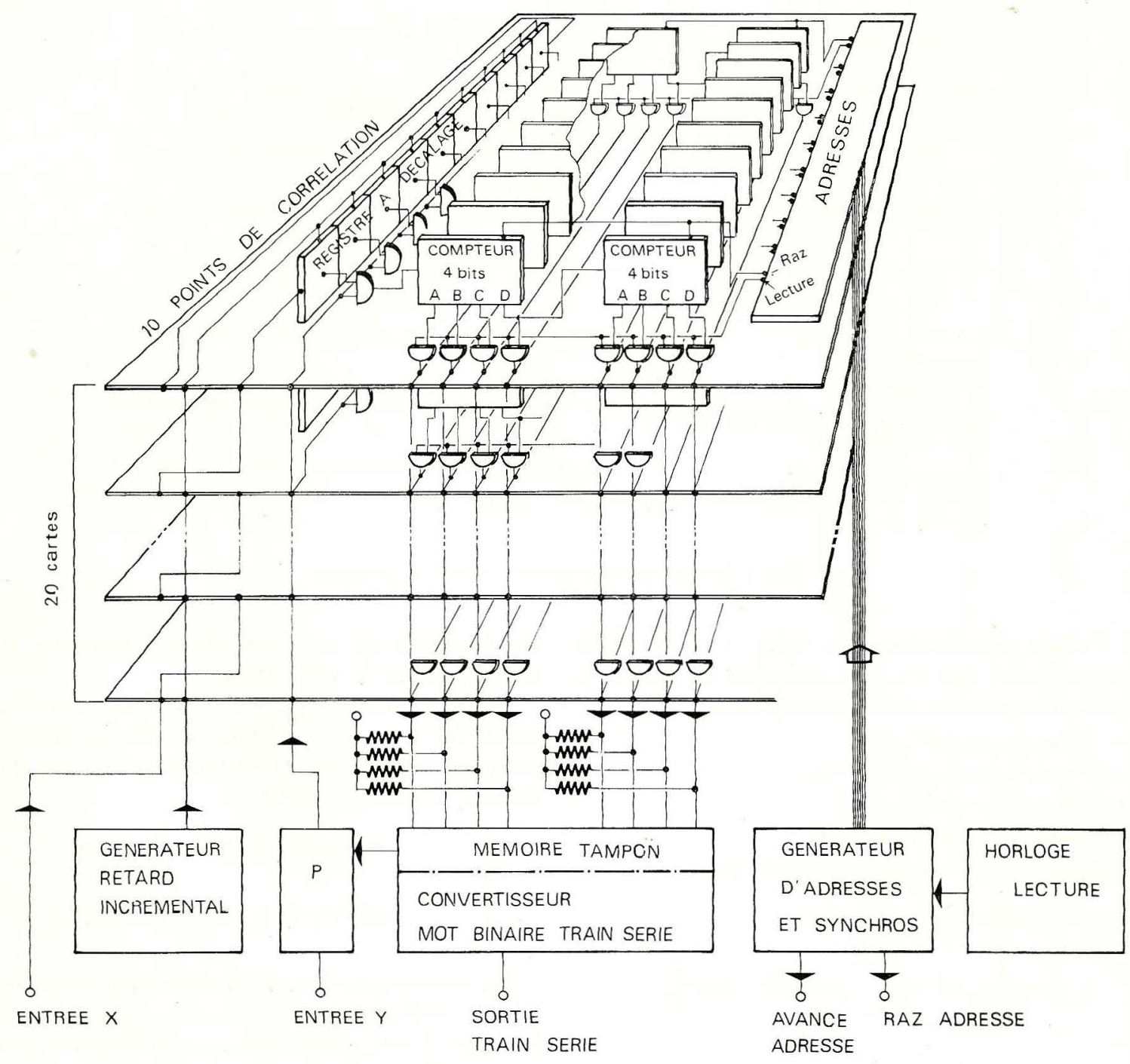

Fig. 3. Schéma logique général.

lecture et le transfert des informations stockées dans chaque canal.

\section{Fonction d'intercorrélation de deux suites d'événements}

L'intérêt de ce corrélateur consiste en la détermination expérimentale de la loi des retards $\tau_{j}$ qui existent entre une suite d'événements mères survenant à des instants $t_{j}$ et une suite d'événements filles survenant aux instants : $s_{j}=t_{j}+\tau_{j}$. Le calcul du coefficient de corrélation relatif à deux fonctions aléatoires du temps liées aux suites $t_{j}$ et $s_{j}$ a été fait de façon exhaustive par Blanc-Lapierre et al. ${ }^{7}$ ). Nous en reprenons les notations et quelques résultats.
Les hypothèses de calcul sont les suivantes :

- la suite $t_{j}$ est stationnaire de densité $\rho_{0}$ constante, - les retards $\tau$ admettent une densité de probabilité $g(\tau)$

- les pertes de comptage se traduisent par une suppression aléatoire des instants $t_{j}$ et $s_{j}$; les probabilités de conserver les instants $t_{j}$ et $s_{j}$ sont respectivement $\alpha$ et $\beta$,

- les fonctions aléatoires relatives aux événements mères et filles sont respectivement :

$$
X(t)=\sum a_{j} Q\left(t-t_{j}\right),
$$

et :

$$
Y(t)=\sum b_{j} P\left(t-t_{j}-\tau\right)
$$



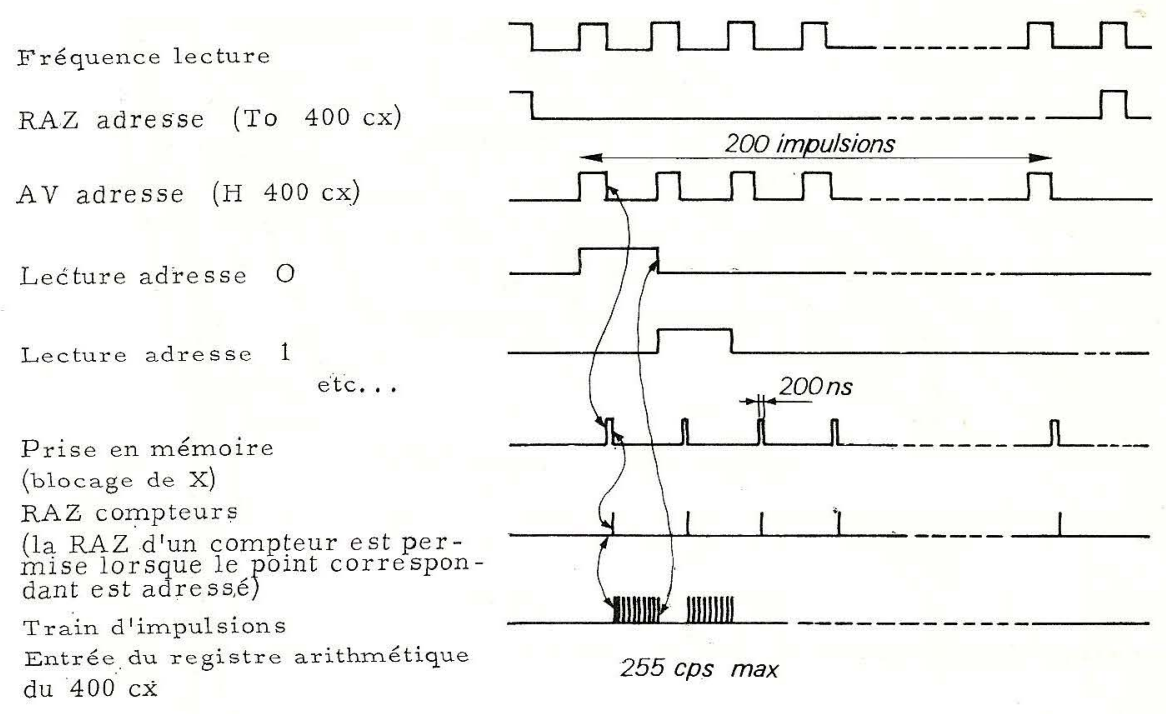

Fig. 4. Diagramme des temps de lecture et transfert.

$Q$ et $P$ étant les réponses des voies $\mathrm{X}$ et $\mathrm{Y}$. Ainsi $Q\left(t-t_{j}\right)=1$ tant que $t-t_{j}$ est inférieur ou égal à la largeur de l'impulsion, et de même pour $P$.

$a_{j}=1$ avec la probabilité $\alpha$;

$a_{j}=0$ avec la probabilité $1-\alpha$;

$b_{j}=1$ avec la probabilité $\beta$;

$b_{j}=0$ avec la probabilité $1-\beta$.

Le coefficient d'intercorrélation s'écrit alors :

$$
\begin{aligned}
C_{\mathrm{XY}}(\lambda) & =E[X(t) Y(t+\lambda)]= \\
& =\rho_{0} \alpha \beta\left[\rho_{0} a b+\int_{-\infty}^{+\infty} g(\tau) c(\lambda-\tau) \mathrm{d} \tau\right],
\end{aligned}
$$

avec :

$$
a=\int_{-\infty}^{+\infty} Q \mathrm{~d} t, \quad b=\int_{-\infty}^{+\infty} P \mathrm{~d} t
$$

et :

$$
c(\lambda)=\int_{-\infty}^{+\infty} Q(\mu) P(\mu-\lambda) \mathrm{d} \mu .
$$

Expérimentalement $C_{\mathrm{XY}}(\lambda)$ est l'espérance d'incrémentation dans l'échelle correspondant au temps $\lambda$ et cela pendant le retard incrémental $\theta$.

Donc pour une durée de mesure grande $T$ (ergodisme) le contenu du canal $i$ de largeur $\theta$ correspondant au temps $\lambda_{\iota}=i \theta$ convergera vers la valeur

$$
(T / \theta) E\left[X(t) Y\left(t+\lambda_{i}\right)\right]
$$

Sur la voie $\mathrm{X}$ les impulsions $Q$ de largeur $\theta$ sont envoyées à l'entrée du registre à décalage (voir 2.2.).
Sur la voie $\mathrm{Y}$ les impulsions $P$ sont mises en phase et quantifiées sur la même durée $\theta$.

Ces conditions expérimentales nous conduisent à écrire les résultats ci-dessus de façon discrète, les fonctions $P(t)$ et $Q(t)$ étant remplacées par le symbole de Kronecker $\delta_{i, 0}$. Donc

$$
\begin{aligned}
& a=b=\sum_{i} \delta_{i, 0} \theta=\theta, \quad \text { et } \\
& c(i \theta)=\sum_{k} \delta_{k, 0} \delta_{k, i} \theta=\theta \delta_{i, 0} .
\end{aligned}
$$

Par suite la représentation théorique de la fonction d'intercorrélation expérimentale accumulée pendant le temps $T$ sera, à condition que la fonction $g(t)$ varie peu sur l'intervalle $\theta$ :

$$
C_{X Y}^{\exp }(i \theta)=\left(T \rho_{0} \alpha \beta / \theta\right)\left[\rho_{0} \theta^{2}+\sum_{k} g(k \theta) \theta \delta_{k, i} \theta\right],
$$

donc :

$$
C_{\mathrm{XY}}^{\exp }(i \theta)=T \rho_{0} \alpha \beta \theta\left[\rho_{0}+g(i \theta)\right] .
$$

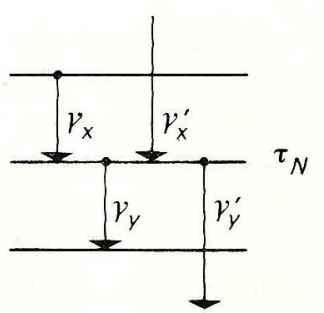

Fig. 5. Alimentation et décroissance d'un niveau nucléaire. 
Les comptages $C_{X Y}^{\exp }(i \theta)$ permettent de dégager simplement la loi $g(\tau)$ qui peut être la caractéristique d'une filiation radioactive, d'un spectre de temps de vol ou de temps de transit ${ }^{7}$ ).

\section{Application - étude d'une filiation radioactive}

La méthode peut être appliquée à la mesure de la vie moyenne $\tau_{\mathrm{N}}$ d'un niveau nucléaire excité.

\subsection{Procédure eXPérimentale}

Dans ce cas les instants $t_{j}$ sont les instants d'apparition du niveau intermédiaire de vie moyenne $\tau_{\mathrm{N}}$ (par émission de $\gamma_{\mathrm{X}}$ ou $\gamma_{\mathrm{X}}^{\prime}$ (fig. 5) et les $s_{j}$ sont les instants de décroissance de ce niveau (par émission de $\gamma_{\mathrm{Y}}$ et $\gamma_{\mathrm{Y}}^{\prime}$ ).

$\mathrm{Ce}$ cas vérifie bien les hypothèses précédentes.

Le dispositif expérimental utilisé est schématisé sur la fig. 6. Après sélection en énergie de la radiation mère $\gamma_{\mathrm{X}}$ les impulsions $Q$ de largeur $\theta$ sont envoyées sur l'entrée $\mathrm{X}$. De même, après sélection des radiations $\gamma_{Y}$ les impulsions $P$ sont mises en phase et envoyées sur l'entrée Y (voir 2.3.).

Avec une loi de filiation radioactive $g(\tau)=\left(1 / \tau_{\mathrm{N}}\right) \times$ $x \exp \left[-\tau / \tau_{N}\right]$, la relation (1) devient :

$C_{\mathrm{XY}}^{\exp }(i \theta)=T \rho_{0}^{2} \alpha \beta \theta+\left(T \rho_{0} \alpha \beta \theta / \tau_{\mathrm{N}}\right) \exp \left[-i \theta / \tau_{\mathrm{N}}\right]$.

\section{Remarques}

La sélection en énergie laisse passer des impulsions étrangères aux deux rayonnements de la cascade nucléaire (Compton, impulsions parasites). Néanmoins l'équation (2) reste valable. Il suffit en effet de considérer qu'une impulsion étrangère dans la voie $X$ est un $\gamma_{X}$ dont le $\gamma_{Y}$ correspondant n'a pas été détecté et inversement. Ce fait expérimental est pris en compte sans aucune restriction par les coefficients $\alpha$ et $\beta$.

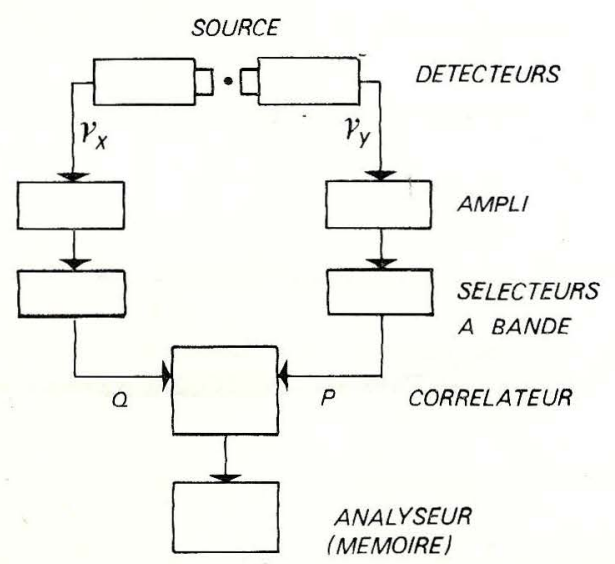

Fig. 6. Schéma de l'appareillage utilisé pour la mesure de $\tau_{\mathrm{N}}$. $\rho_{0}$ représente alors une densité fictive de formation de l'état intermédiaire, supérieure à la densité réelle. $\alpha$ et $\beta$ sont donc des efficacités fictives définies par $\rho_{0} \alpha=I_{\mathrm{X}}, \rho_{0} \beta=I_{\mathrm{Y}}, I_{\mathrm{X}}$ et $I_{\mathrm{Y}}$ étant les intensités réelles à l'entrée du corrélateur.

Par suite $\alpha$ et $\beta$ tiennent compte des efficacités de détection, des angles solides, des bruits de fond et des rapports d'alimentation et de branchement $=$ $=I_{\mathrm{X}} /\left(I_{\mathrm{X}}+I_{\mathrm{X}}^{\prime}\right)$ et $I_{\mathrm{Y}} /\left(I_{\mathrm{Y}}+I_{\mathrm{Y}}^{\prime}\right)$.

L'équation (2) peut être interprétée de la façon suivante : $T \rho_{0}^{2} \alpha \beta \theta$ représente le nombre de coïncidences accidentelles accumulées pendant le temps $T$ par un circuit de coïncidences de résolution $\theta$, et $T \rho_{0} \alpha \beta$ est le nombre de coïncidences vraies entre $\gamma_{\mathrm{X}}$ et $\gamma_{Y}$ donné par un circuit de coïncidence de temps de résolution bien supérieur à $\tau_{\mathrm{N}}$. $C_{\mathrm{XY}}^{\exp }(\lambda)$ peut donc décrire la courbe de corrélation donnée par un convertisseur temps-amplitude classique dans le cas limite où le temps de blocage de celui-ci est très inférieur au temps moyen séparant deux entrées.

\subsection{EXPloitation des RÉSUltats, PRÉCISION}

Le comptage étant de type poissonnien, la variance du contenu $C_{i}$ du canal $i$ est :

$$
\sigma_{C_{i}}^{2}=C_{i}
$$

Nous utilisons la méthode des moindres carrés pour lisser la courbe expérimentale par une fonction de la forme :

$$
C_{i}=N_{\mathrm{FC}}+N_{\mathrm{v}} \frac{\theta}{\tau_{\mathrm{N}}} \exp \left[-i \theta / \tau_{\mathrm{N}}\right],
$$

dans laquelle $N_{\mathrm{FC}}$ est le nombre de coincïdences fortuites par canal et $N_{\mathrm{v}}$ le nombre de coincïdences vraies entre les raies $\gamma_{\mathrm{X}}$ et $\gamma_{\mathrm{Y}}$.

On a :

$$
N_{\mathrm{FC}}=T \rho_{0}^{2} \varepsilon^{2} \theta
$$

et :

$$
N_{\mathrm{v}}=T \rho_{0} \varepsilon^{2}
$$

avec :

$$
\varepsilon^{2}=\alpha \beta .
$$

$\varepsilon$ représente l'efficacité moyenne sur les deux entrées. De même, l'intensité moyenne est :

$$
I=\left(I_{\mathrm{X}} I_{\mathrm{Y}}\right)^{\frac{1}{2}} \text {. }
$$

Les variances sur les paramètres $N_{\mathrm{FC}}, N_{\mathrm{v}}$ et $\tau_{\mathrm{N}}$ sont données par les éléments diagonaux de l'inverse de la matrice normale relative à la méthode des moindres 
carrés $\left.^{8}\right)$. L'erreur relative $\Delta \tau_{N} / \tau_{N}$ est de la forme :

$$
\frac{\Delta \tau_{\mathrm{N}}}{\tau_{\mathrm{N}}}=\frac{\Phi\left(\rho_{0}, \tau_{\mathrm{N}}, N, \theta\right)}{\varepsilon(T)^{\frac{1}{2}}} .
$$

$\Phi$ est une fonction à calculer numériquement et $N$ représente le nombre de canaux. En fait $\tau_{\mathrm{N}}, N$ et $\theta$ sont liés entre eux par l'étalonnage en temps du dispositif expérimental, $N \theta$ étant la gamme d'analyse. Nous allons introduire le paramètre $x=N \theta / \tau_{N}$ qui représente le nombre de vies moyennes contenues dans cette gamme.

Les abaques des figs. 7 et 8 montrent l'évolution de $\Phi$ en fonction des paramètres $\rho_{0}, \tau_{\mathrm{N}}, x$ et $N$. On peut voir (fig. 7) que pour $\rho_{0}>10000 \mathrm{c} / \mathrm{s}$, la précision tend rapidement vers une limite. $x=6$ semble être une bonne valeur de travail et il n'est pas nécessaire d'avoir un nombre de canaux supérieur à 200 .

\subsection{Mesure de La Vie moyenne du niveau $7^{-}$ EXCITÉ À $2200 \mathrm{keV}$ DANS LE ${ }^{206} \mathrm{~Pb}$}

Afin d'illustrer l'intérêt de la méthode pour les vies moyennes dans le domaine $1 \mu \mathrm{s}-1 \mathrm{~s}$, nous avons remesuré la vie moyenne $\tau_{\mathrm{N}}$ du niveau $7^{-}$excité à $2200 \mathrm{keV}$ dans $1 \mathrm{e}^{206} \mathrm{~Pb}$.

Nous avons préparé la source de ${ }^{206} \mathrm{Bi}$ qui alimente les niveaux excités $\mathrm{du}{ }^{206} \mathrm{~Pb}$ par décroissance $\beta^{-9}$ )

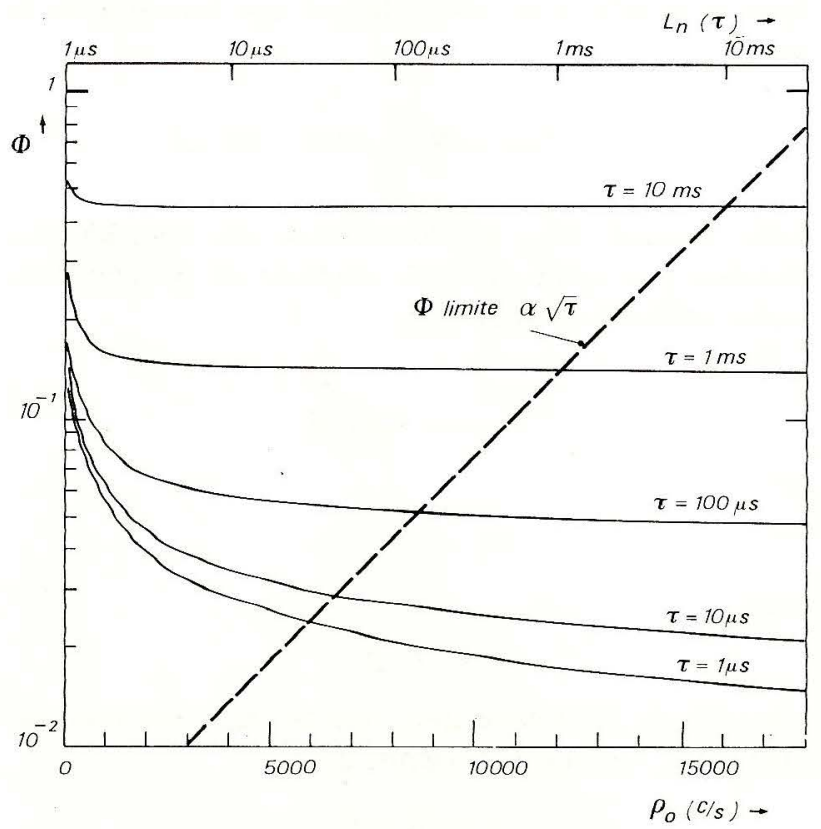

Fig. 7. Variation de la grandeur numérique $\Phi$ en fonction du taux de comptage $\rho_{0}$ et de la vie moyenne $\tau_{N}$ étudiée. La courbe donnant $\Phi_{1 \mathrm{imite}}$ est relative à l'échelle supérieure $\log \tau_{\mathrm{N}}(x=5$, $N=200$ ). sur irradiation de thallium au synchrocyclotron de Lyon : ${ }^{203} \mathrm{Tl}(\alpha, \mathrm{n})$ et ${ }^{205} \mathrm{Tl}(\alpha, 3 \mathrm{n})$.

Nous avons sélectionné la raie de $184 \mathrm{keV}$ qui alimente le niveau et celle de $516 \mathrm{keV}$ qui le désexcite. Ces radiations gamma étaient détectées à l'aide d'ensembles photomultiplicateurs-scintillateurs $\mathrm{NaI}(\mathrm{Tl})$. Afin d'éviter l'influence des rétrodiffusions au niveau du pic $184 \mathrm{keV}$, nous avons utilisé une géométrie pour laquelle les efficacités n'étaient que $\varepsilon=2,2 \times 10^{-2}$. Les mesures ont été effectuées 4 jours après l'irradiation pour éliminer les isotopes ${ }^{203} \mathrm{Bi}$ et ${ }^{204} \mathrm{Bi}$.

Nous avons obtenu la valeur $\tau_{N}=(170 \pm 3) \mu \mathrm{s}$. Cette valeur est en accord avec le résultat de Tove ${ }^{10}$ ).

\section{Avantage de la methode}

Nous comparerons ici les principales méthodes utilisées pour mesurer les vies moyennes.

\subsection{Convertisseur temps-Amplitude (C.T.A.)}

Le C.T.A. effectue le classement des intervalles de temps séparant deux impulsions mère et fille. En l'absence de filiation, la courbe expérimentale converge vers l'exponentielle caractéristique de la loi des intervalles de temps dans une suite poissonnienne de densité $2 I$. Dans le canal de rang $i$, le nombre de coïncidences fortuites est proportionnel à $2 I \exp [-2 I i \theta]$, la variation relative du fond de coincidences fortuites $N_{\text {FC }}$ est alors

$$
\left(\Delta N_{\mathrm{FC}} / N_{\mathrm{FC}}\right)=2 I N \theta=2 \rho_{0} \varepsilon N \theta .
$$

Dans la gamme $10^{-5} \mathrm{~s}<N \theta<1 \mathrm{~s}$, ce rapport peut devenir suffisamment important pour entrainer une erreur systématique.

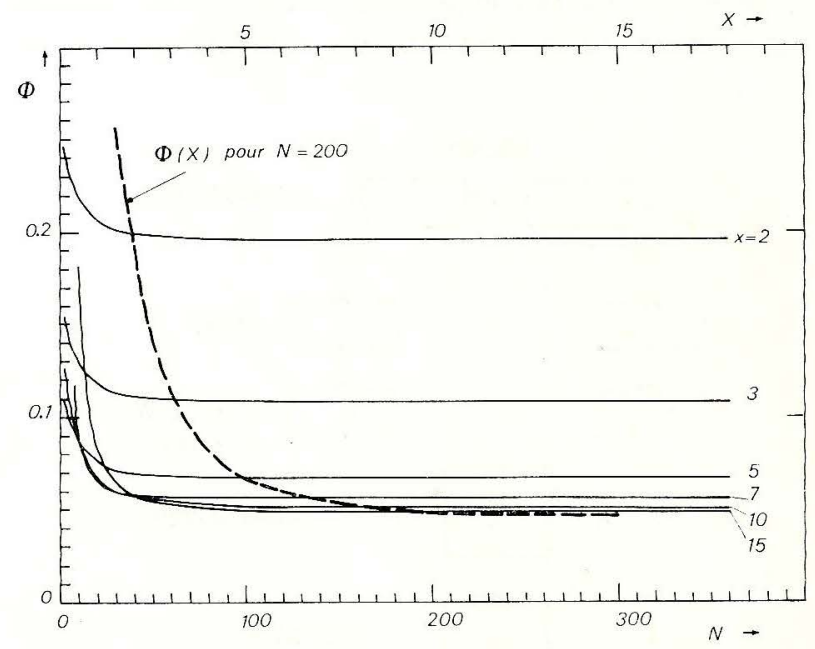

Fig. 8. Variation de $\Phi$ en fonction du nombre de canaux utilisés $N$ pour différents étalonnages $x\left(\tau_{\mathrm{N}}=200 \mu \mathrm{s}, \rho_{0}\right.$ grand $)$. 
TABleaU 1

Comparaison des temps de comptage pour les trois techniques dans les conditions de notre mesure de $\tau_{\mathrm{N}}\left(7^{-},{ }^{206} \mathrm{~Pb}\right) . \quad(\varepsilon=$ $\left.=2,2 \times 10^{-2} ;(\Delta \tau / \tau)=1,5 \% ; \rho_{0}=23000 \mathrm{c} / \mathrm{s}\right)$.

\begin{tabular}{llll}
\hline & C.T.A. & Codeur en temps & Corrélateur \\
\hline \multirow{2}{*}{$\tau_{\mathrm{N}}=170 \mu \mathrm{s}$} & $T=40 \mathrm{~h}$. & $T=14 \mathrm{~h}$. & $T=9 \mathrm{~h}$. \\
& $\rho_{0}=390 \mathrm{c} / \mathrm{s}$ & $\rho_{0}=23000 \mathrm{c} / \mathrm{s}$ & $\begin{array}{l}\rho_{0}=23000 \mathrm{c} / \mathrm{s} \\
\tau_{\mathrm{N}}=1 \mathrm{~ms}\end{array}$ \\
& $T=9 \mathrm{j}$. & $T=7,3 \mathrm{j}$. & $T=2 \mathrm{j}$. \\
& $\rho_{0}=70 \mathrm{c} / \mathrm{s}$ & $\rho_{0}=23000 \mathrm{c} / \mathrm{s}$ & $\rho_{0}=23000 \mathrm{c} / \mathrm{s}$ \\
\hline
\end{tabular}
si:

On peut considérer cette erreur comme négligeable $\left(\Delta N_{\mathrm{FC}} / N_{\mathrm{FC}}\right)<\frac{1}{10}\left(\sigma N_{\mathrm{FC}} / N_{\mathrm{FC}}\right)$ (fluctuation statistique).

Cette condition s'écrit :

$$
20 \rho_{0} \varepsilon N \theta<\left(\rho_{0} \varepsilon\right)^{-1}(T \theta)^{-\frac{1}{2}},
$$

c'est-à-dire :

$$
\rho_{0}<\varepsilon^{-1}(20 N \theta)^{-\frac{1}{2}}(T \theta)^{-\frac{1}{4}}
$$

Les relations 3 et 4 définissent les valeurs extrêmes de $\rho_{0}$ et $T$ pour atteindre une précision donnée avec le C.T.A. Ces valeurs sont calculées dans deux cas particuliers de vie moyenne et portées dans le tableau 1.

\subsection{Codeur en temps (SLOW time AnAlyser)}

Dans ce cas l'impulsion mère déclenche un balayage d'ouverture de chaque canal d'un analyseur multiéchelle pendant le temps $\theta$, l'impulsion fille incrémente le canal ouvert à l'instant où elle se présente. En l'absence de filiation, la fonction de corrélation est parfaitement horizontale.

Une limite à l'utilisation de cet appareil provient de la perte d'événements mères pendant la durée du balayage $N \theta$. Si $I_{x}$ est l'intensité vraie, l'intensité réellement prise en compte est :

$$
I_{x}^{\prime}=I_{x}\left(1+I_{x} N \theta\right)^{-1} \text {. }
$$

L'équation (2) peut représenter le fonctionnement de cet appareil en remplaçant $\alpha$ par $\alpha^{\prime}=\alpha\left(1+\rho_{0} \alpha N \theta\right)^{-1}$ et $\varepsilon$ par $\varepsilon^{\prime}=\varepsilon(1+I \varepsilon N \theta)^{-\frac{1}{2}}$.

A précision égale, le temps de mesure devient :

$$
T^{\prime}=T\left(1+I_{x} N \theta\right)
$$

nous comparons dans le tableau 1 les temps de mesure nécessaires pour atteindre une même précision avec ces différentes techniques.

\subsection{Corrélateur de Landaud}

La méthode de Landaud $^{4,5}$ ) consiste à étudier la fonction d'autocorrélation de la réponse d'un bistable aux impulsions d'un détecteur unique acceptant à la fois les événements mère et fille.

Pour 200 points, la précision de cette méthode est comparable sur le plan temporel à celle de l'intercorrélation bien que le traitement mathématique soit plus délicat. Ce système ne permet pas par contre d'atteindre le nombre de coïncidences vraies, cette information est très utile dans le cas de la mesure d'une corrélation angulaire par exemple.

La technique de mesure que nous avons présentée ici s'inscrit dans le cadre général de la méthode des coïncidences retardées. Dans tous les cas elle nous conduit (aux fluctuations statistiques près) à une courbe de corrélation exacte alors que dans ce même cadre un convertisseur temps-amplitude ne donne que des résultats approchés. Notons de plus que cette technique peut également être utilisée pour des mesures de corrélations angulaires. Nous poursuivons actuellement des études dans ce sens.

Nous tenons à remercier MM. les Professeurs I. Berkes et J. Depraz pour les nombreuses et fructueuses discussions que nous avons eues avec eux tout au long de ce travail.

\section{Références}

1) J. Max, Méthodes et techniques de traitement du signal et applications (Editions Masson; Paris, 1972).

2) R. Billerey, D. Charnay, J. Depraz et E. Descroix, Spectrométrie neutronique par intercorrélation (LYCEN-7262) (1972).

3) M. Rotival, Thèse de spécialité (Lyon, 1970).

4) G. Landaud, Nucl. Instr. and Meth., 26 (1964) 117.

5) D. Favret, Thèse Doct. spécialité (Lyon, 1969).

6) D. Charnay, H. Chevalier et J. P. Gorius, Rapport Annuel Institut de Physique Nucléaire de Lyon (LYCEN-7301) p. 52.

7) A. Blanc-Lapierre et P. Dumontet, C.R.A.S., 250A (1960) 1216.

8) Y. V. Linnik, Méthode des moindres carrés (Ed. Dunod; Paris 1963).

$\left.{ }^{9}\right)$ M. Lederer, J. M. Hollander and I. Perlman, Table of isotopes (Wiley and Sons, New York, 1967).

10) P. A. Tove, Nucl. Instr. and Meth. 1 (1957) 95. 